\title{
Growth, study, and device application prospects of graphene on $\mathrm{SiC}$ substrates
}

\author{
A. A. Lebedev \\ Ioffe Institute, St. Petersburg, Russia \\ Shura.lebe@mail.ioffe.ru
}

PACS 61.48.Gh, 73.22.Pr

DOI 10.17586/2220-8054-2016-7-1-30-36

\begin{abstract}
It is well known that graphene, which brought the 2010 Noble Prize in physics to Russian scientists Andre Geim and Konstantin Novoselov, exists as two-dimensional carbon layers. The possible range of graphene applications includes development of field-effect transistors for digital and analog electronics, nanoelectromechanical systems, quantum dots, cold cathodes, supersapacitor, gas sensors, and nearly transparent electrodes and coatings. The possibility of using graphene for hydrogen storage and the manufacture of composite materials is also being studied. Being a two-dimensional material, graphene provides ultimate one-dimensional miniaturization and is a convenient basis for manufacture of different nanoelectronic, nanomechanical and nanochemical devices by lithographic methods. The graphene-based device which is closest to being successfully realized for practical application is the gas sensor. The use of graphene makes it possible to achieve a sensitivity exceeding that of all other materials, less than $1 \mathrm{ppb}$. This device combines the comparative simplicity of manufacture with a wide spectrum of possible applications. It should also be mentioned that the structure of the gas sensor actually reproduces the structure of the field-effect transistor. Thus, the gas sensor can be considered the first stage in the development of intricate transistor electronics based on graphene. The paper briefly reviews growth experiments and studies of graphene films on silicon carbide $(\mathrm{SiC})$ and the development of prototype gas sensors based on this material.
\end{abstract}

Keywords: graphene, silicon carbide, sublimation, gas sensors.

Received: 20 November 2015

\section{Introduction}

Graphene is a promising material that has unique properties, such as high surfaceto-volume ratio, low electrical noise, and exceptional transport properties associated with its two-dimensional structure [1]. After a graphene monolayer was isolated in 2004 [1], much effort has been exerted to study the fundamental properties of this unique structure [2-6]. The most important of these is related to the confinement of carrier motion, which results in nearly linear carrier energy dispersion and, as a consequence, in extremely small effective mass and huge carrier mobility along the $2 \mathrm{D}$ graphene plane

Recently, one of the popular methods used to form graphene layers has been the thermal graphitization of single-crystal silicon carbide in a vacuum or under an argon atmosphere [5]. In contrast to the standard approaches, this method can be used to fabricate large graphene samples and allows further lithographic processing. The method utilizes the non-stoichiometric sublimation of silicon from the surface of single-crystal $\mathrm{SiC}$ heated to high temperatures, which yields a hexagonal lattice of carbon atoms remaining on its surface. The quality of the layers being obtained is provided by a pre-growth treatment of the $\mathrm{SiC}$ substrate. Note that, as far as we know, this method most probably gives a multilayered structure. Thus, we believe that a study of such structures is an important issue for the physics and technology of graphene. 
The high adsorption capacity and the large surface-to-volume ratio of graphene make it attractive as a gas sensing material. In recent years, the interest in air pollutants and their monitoring has steadily increased. Nitrogen dioxide $\left(\mathrm{NO}_{2}\right)$ is a typical air pollutant that causes environmental and health problems. From this perspective, it is necessary to develop a highly sensitive and an inexpensive gas sensor capable of detecting low concentrations of this gas. Currently, gas sensing experiments demonstrate that epitaxial graphene may be an excellent material for future $\mathrm{NO}_{2}$ sensors. Graphene layers grown on $\mathrm{SiC}$ have demonstrated sensitivities down to the ppb level and a high selectivity for $\mathrm{NO}_{2}$ detection in the presence of the typical interfering gases $[7,8]$.

\section{Sublimation growth of graphene}

It is well known that heating of silicon carbide ( $\mathrm{SiC}$ ) causes the primary sublimation of silicon ( $\mathrm{Si}$ ) atoms and the graphitization of the underlying silicon carbide surface [9] (Fig. 1). After the discovery of numerous forms of nanocarbon (nanotubes, fullerenes, etc.) there appeared an idea of obtaining new carbon structures on silicon carbide via the in vacuo-controlled thermal decay of the crystal's surface $[10,11]$.

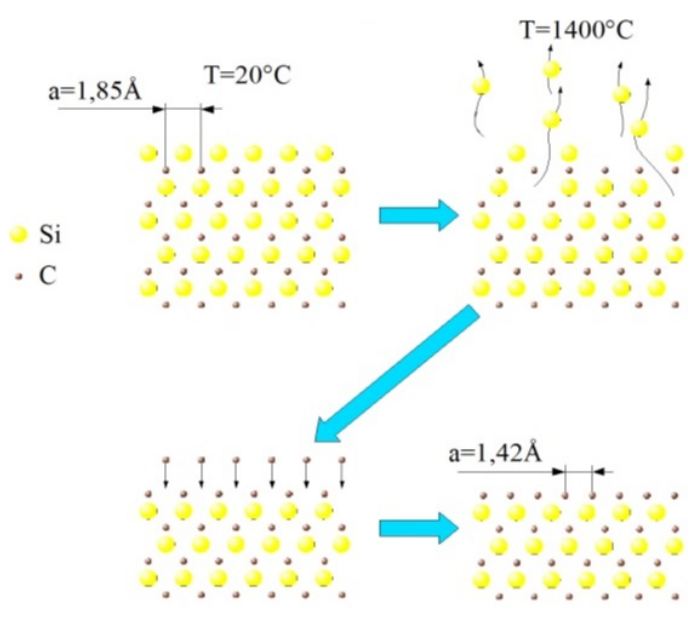

FIG. 1. Diagram of how graphene is formed on the surface of a SiC wafer

After the first planar nanocarbon films ("exfoliated grapheme") were obtained in 2004 by the laser-mediated peeling from bulk crystalline graphene [1], studies were focused on obtaining a monoatomic layer of carbon on a $\mathrm{SiC}$ wafer. There have been several publications reporting that graphene films were obtained on the $\mathrm{SiC}$ surface. The communications $[12,13]$ demonstrated that a graphene film can be obtained in a high vacuum $\left(\sim 10^{-10}\right.$ Torr $)$ on axis $6 \mathrm{H}$-SiC wafer by the surface thermal decay method. In [14], planar monoatomic carbon films formed on a $4 \mathrm{H}$ silicon carbide wafer were also studied.

It was shown in $[15,16]$ that nanocarbon layers can be formed on the $\mathrm{SiC}$ surface by the sublimation method in an open system. At the same time, no additional etching of samples in hydrogen or silicon vapors was required. This significantly simplified the process by which the films were obtained. The thickness of the resulting film was strongly dependent upon the annealing temperature. However, the structural and electrical properties of this film were more variable and sensitive to numerous technological factors, including the pre-growth surface treatment. Some of the existing dependences have been determined, but further analysis of these is required. According to the reflection high-energy electron diffraction (RHEED) method, the film contained two-dimensional graphene crystals. Measurements demonstrated that the films 
formed on the carbon face of $\mathrm{SiC}$ have a better structural perfection than those formed on the silicon face. The data obtained confirm that the optimal temperature range to form graphene on the $\mathrm{SiC}$ surface is $1300-1400{ }^{\circ} \mathrm{C}$.

\section{Analysis of the films obtained}

In addition to the conditions in which planar nanocarbon layers are formed on the $\mathrm{SiC}$ surface, various properties of the carbon film and the graphite/silicon carbide system are also being actively studied. The authors of [17] examined the electronic structure of the interface between the $6 \mathrm{H} \mathrm{SiC} \mathrm{(0001)} \mathrm{wafers} \mathrm{and} \mathrm{the} \mathrm{graphite} \mathrm{film.} \mathrm{A} \mathrm{structural} \mathrm{analysis} \mathrm{of} \mathrm{the}$ multigraphene (several graphene layers) structure/4H-SiC was made in [18]. The samples were subjected to a surface $X$-ray diffraction analysis. The distance between the wafer and the first monoatomic graphene layer was shown to be only $1.6 \AA$.

However, in all of the above-mentioned articles, attention was given to the structural analysis of graphene films, without due consideration of their electrical properties. This is partly due to the difficult fabrication of any contacts to such nano-objects as a one-layer-thick carbon film. A technological process for the manufacture of a graphene-based MESFET was suggested in [19]. The only exception is the communication [20], in which the formation of a field transistor structure on a film grown on the $\mathrm{SiC}$ surface was reported. As follows from the text of this communication, the carrier mobility in the given film was $535 \mathrm{~cm}^{2} /(\mathrm{V} \cdot \mathrm{s})$, which is considerably lower than the mobility in the "exfoliated" graphene, where, according to [2], it has a value of $15,000 \mathrm{~cm}^{2} /(\mathrm{V} \cdot \mathrm{s})$ at room temperature.

A possible reason for this behavior is the low structural perfection of the graphene film studied in [21], which, according to the AFM image, had multiple holes $\sim 0.5 \mu \mathrm{m}$ in diameter. One possible reason why the film is porous is the imperfection of the pre-growth surface treatment, including residual surface impurities (oxygen and other adsorbates), which can react with carbon atoms during the course of thermal destruction to form volatile components (e.g. $\mathrm{CO}, \mathrm{CO}_{2}$ ). Detailed analyses have been made of Raman spectra of the films [22] and their transport properties [23].

In $[24,25]$, test structures for electrical measurements were formed the sample surface in the Hall-bar configuration by etching with an argon beam through a photoresist mask. Measurements of the current-voltage characteristics and the Hall effect were made at temperatures ranging from $2-300 \mathrm{~K}$. It was stated that at $T<4 \mathrm{~K}$, the conductivity weakly depends on temperature and becomes lower with decreasing temperature, in accordance with the weak logarithmic law typical of two-dimensional dirty metals (weak localization mode). According to the theory, the slope ratio of the logarithmic dependence for a two-dimensional system is universal and defined as $e^{2} / h \sim 4 \cdot 10^{-5} \mathrm{Ohm}^{-1}$. For the samples under study, this slope ratio is almost the same $\left(2-3 \cdot 10^{-6}\right)$, which is several times less than the universal value. However, the conductivity calculations disregarded the following fact: the samples had a rectangular, rather than square, shape. The resistance for the square should then be reduced by a factor of $5-10$. Accordingly, the slope ratio for the conductivity temperature dependence should be increased (at the sample length 10 times the width, this slope ratio will be $2 \cdot 10^{-5} \mathrm{Ohm}^{-1}$, which almost coincides with the theoretical estimation). At low temperatures $(1.4 \mathrm{~K})$, the conductivity tends to level-out ("saturate") for both samples. The observed saturation of the low-temperature conductivity can be attributed to the finite size of the conducting sample $(\sim 10 \mu \mathrm{m})$, compared to the phase fault length $L$, which at low temperatures may be comparable with the sample size. At the same time, the logarithmic dependence disappears. Multigraphene is characterized by $L$ of $\sim 1-10 \mu \mathrm{m}$ at low $T$. The Hall effect measured at low temperatures, gives for all temperatures the same carrier concentration $n \sim 10^{12} \mathrm{~cm}^{-2}$ for one layer. At the same time, the Hall mobility 
is low, about $100 \mathrm{~cm}^{2} /(\mathrm{V} \cdot \mathrm{s})$. The comparatively high carrier concentration $\left(10^{12} \mathrm{~cm}^{-2}\right)$ and low mobility, $100 \mathrm{~cm}^{2} /(\mathrm{V} \cdot \mathrm{s})$, confirmed that, rather than being intrinsic, carriers are due to defects in multigraphene or at the SiC-multigraphene interface $(\mathrm{GR} / \mathrm{SiC})$.

In [25], the properties of a graphene film grown on $\mathrm{SiC}$ were studied "ex situ" by atomic force microscopy (AFM), Raman spectroscopy (Fig. 2), low energy electron diffraction (LEED), $X$-ray photoelectron spectroscopy (XPS), and near edge x-ray absorption fine structure (NEXAFS) spectroscopy. The AFM study showed that the substrate surface consisted of flat and wide $(\sim 1 \mu \mathrm{m})$ terraces covered with sufficiently large and continuous graphene domains. Numerous LEED patterns obtained from different points of the sample demonstrated the concurrent presence of a well-ordered graphite $(1 \times 1)$ pattern and $(6 \sqrt{3} \times 6 \sqrt{3}) \mathrm{R} 30$ pattern inherent to the underlying buffer layer [26], thus confirming the mainly bilayer nature of the film.

XPS and NEXAFS data obtained on a BESSY II synchrotron (Berlin) specify the chemical composition and electronic structure of the graphene film and confirm its high perfection and mostly bilayer nature. In particular, the bilayer character of the film was confirmed by low energy electron diffraction experiments, as well as by the $0.2 \mathrm{eV}$ shift of the spectrum due to the charge transfer to the substrate, which is typical for bilayer films [27-29]. Considering the bilayer as a quantum well with a $\mathrm{SiC}$ bandgap wall, one can assume that the van Hove singularities appear in the density of states because of the quantum confinement in the normal direction. Indeed, two peaks are seen in the valence band spectrum of graphene near the Fermi level. Their energies are $E_{1}=0.5-0.2=0.3 \mathrm{eV}$ and $E_{2}=1.5-0.2=1.3 \mathrm{eV}$, with consideration for the $0.2 \mathrm{eV}$ spectrum shift due to the charging the layer. The energy ratio $\left(E_{2} / E_{1} \sim 4=n_{1} / n_{2}\right)$ and values agree with those calculated for the quantum well (Figs. 3, 4).

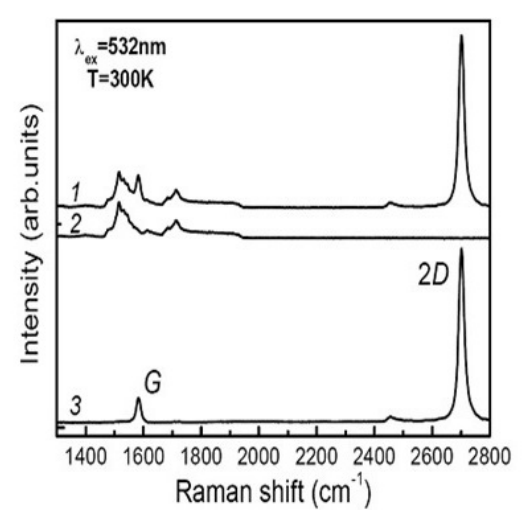

FIG. 2. 1 - Raman spectrum of a graphene layer grown on a $6 \mathrm{H}-\mathrm{SiC}$ substrate; 2 - secondorder Raman spectrum of the substrate $6 \mathrm{H}-\mathrm{SiC} ; 3$ - differential spectrum obtained by subtracting spectrum 1 from spectrum 2

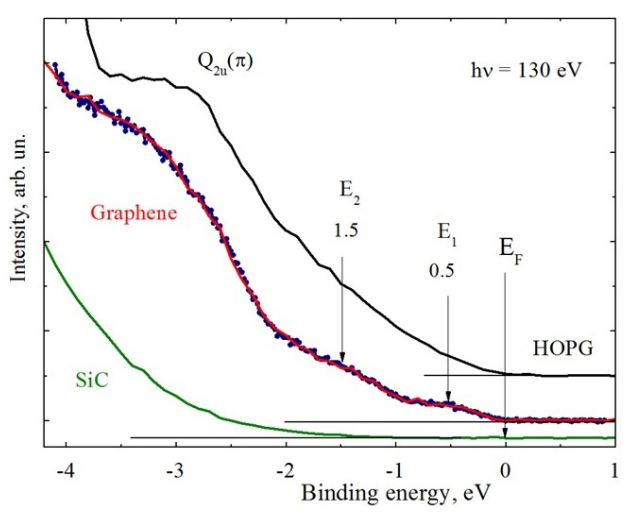

FIG. 3. Valence-band densityof-states spectra of the $\mathrm{SiC}$ substrate, bilayer graphene film, and pyrolytic graphite

\section{Graphene gas sensors}

Currently, there is an increasing demand for a cost-effective sensor for the detection of environmental pollutants like $\mathrm{NO}_{x}$. Traditionally, transition metal oxides, such as $\operatorname{In}_{2} \mathrm{O}_{3}$, $\mathrm{SnO}_{2}, \mathrm{ZnO}$, and $\mathrm{WO}_{3}$, have been used for sensing [30]. However, a serious drawback for 
these oxides is that their functional characteristics are strongly dependent upon the conditions used in their syntheses. Recently, epitaxial graphene on $\mathrm{SiC}$ has been suggested as a promising route for the mass-production of uniform, wafer-size graphene layers for the detection of gas molecules [7]. At present, this technique can give very high quality graphene films, the size of which is basically limited by the size of the $\mathrm{SiC}$ substrate. This technique also provides integration of graphene-based sensors with high-temperature electronics based on SiC. In this communication, we report the fabrication of sensors based on graphene films and their tests in ambient gas sensing.

Gas sensors were fabricated from the epitaxial graphene films grown by annealing of $\mathrm{SiC}$ substrates under Ar or vacuum at temperatures of $1200-1600{ }^{\circ} \mathrm{C}$. Depending on the annealing time, temperature and atmosphere, the graphene films can contain $1-3$ layers. The thickness of the graphene films was estimated by Auger and Raman spectroscopy. Patterns for the sensor devices were produced on the graphene surface of the substrate by laser photolithography over AZ5214 resist. Reactive ion etching in argon-oxygen plasma was used to remove the graphene layer from uncoated areas. $\mathrm{Ti} / \mathrm{Au}(5 / 50 \mathrm{~nm})$ contacts were formed by $e$-beam evaporation and lift-off photolithography with a laser writer over AZ5214 resist. The sample chip was assembled on the holder together with two Pt100 resistors. One of the resistors was chosen for temperature measurements, while the other served as a heater (Fig. 5).

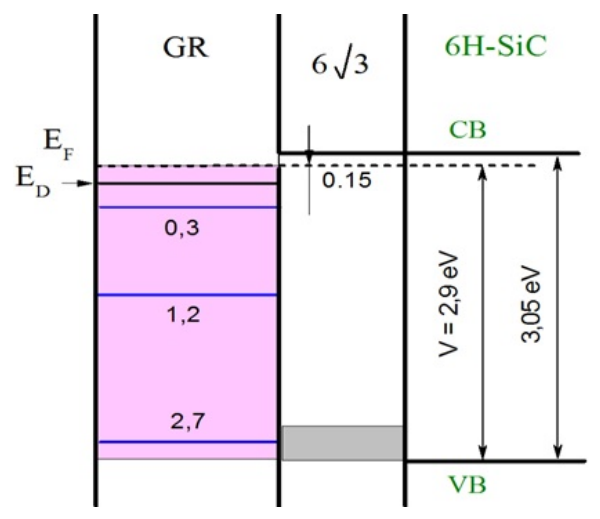

FIG. 4. Scheme of the quantum well for holes, formed on $\mathrm{SiC}$ with $6 \sqrt{3}$ interface carbon monolayer (buffer layer)

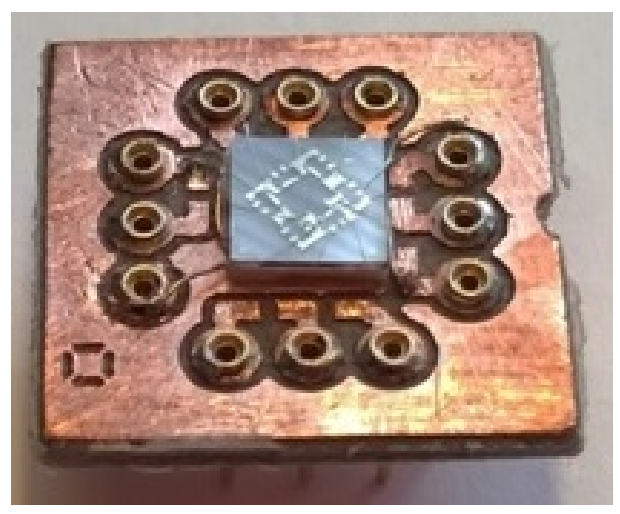

FIG. 5. Sensor device on holder

To measure the gas response of graphene, a custom-made gas system was used. The dilution ratio could be varied from $1: 1-1: 10^{5}$, which provided an output concentration in the range from $0.1 \mathrm{ppb}$ (parts per billion) to $10 \mathrm{ppm}$ (parts per million). The response $r$ was expressed in percent and defined as the relative change in the resistance of the sample upon its exposure to the gas, $r=\left(R-R_{0}\right) / R_{0}$, where $R$ is the resistance with the gas, and $R_{0}$ is the resistance of the graphene film under the initial conditions defined by the air flow.

In Fig. 6, the relative changes in the resistance of graphene-based sensors upon exposure to $\mathrm{NO}_{2}$ (exposure periods marked as light grey bands) at $20{ }^{\circ} \mathrm{C}$ are presented. Because the desorption rate of $\mathrm{NO}_{2}$ is very low at room temperature, the sensor was heated at $110{ }^{\circ} \mathrm{C}$ after each exposure to restore it to its initial pre-measurement state [8].

The response signs for samples grown by different methods are opposite. According to Raman spectroscopic data, the graphene grown by vacuum annealing is multilayer, while that grown under Ar is single-layer. For multilayer graphene, $p$-type conductivity is expected, whereas $n$-type conductivity is observed for the single-layer material. $\mathrm{NO}_{2}$ is a strong oxidizer, 

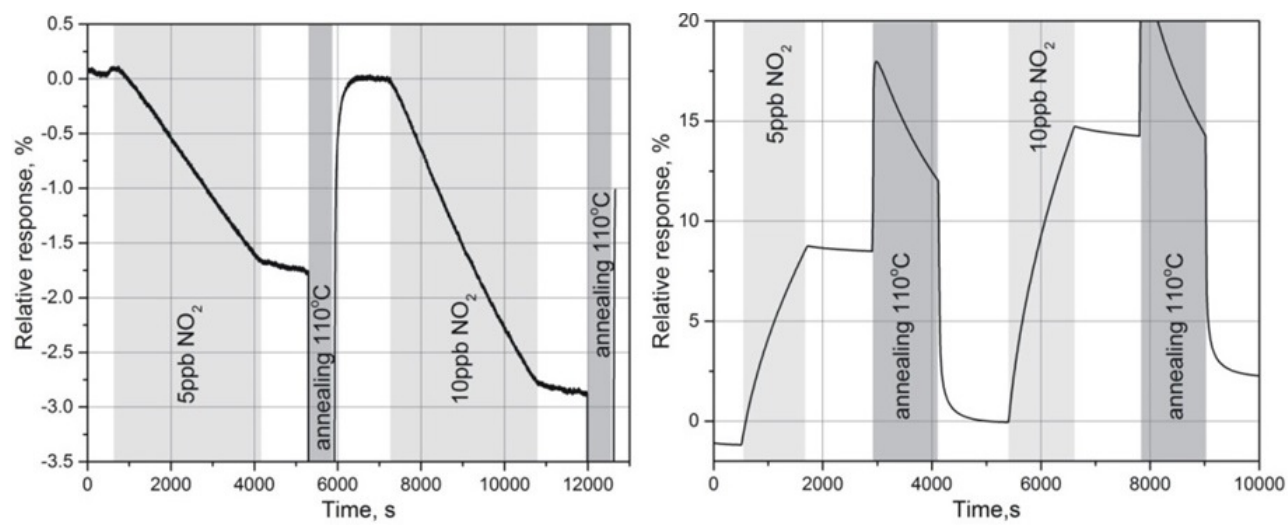

FIG. 6. Response upon exposure of graphene grown by annealing in a vacuum (left) and in argon (right) to a gas mixture containing $\mathrm{NO}_{2}$ gas at $20{ }^{\circ} \mathrm{C}$. The exposure periods are marked as light gray bands, and the annealing periods, as dark gray bands

withdrawing electrons from the surface on which it is adsorbed. Therefore, its adsorption on the graphene surface is expected to reduce the electron density and raise the hole content. In the case of the $n$-type material, this leads to an increase in resistivity, whereas for the $p$-type, the resistivity decreases. For the $\mathrm{NO}_{2}$ concentration of $10 \mathrm{ppb}$, which is typical for environmental monitoring, the response of the multilayer graphene-based sensor is about $3 \%$ upon a $1 \mathrm{~h}$ exposure. The response of the single-layer graphene to $\mathrm{NO}_{2}$ exposure is much stronger and reaches $15 \%$ under the same conditions. This can be accounted for by the screening of the inner layer of the multilayer graphene from adsorbed $\mathrm{NO}_{2}$ molecules. Nevertheless, the sensitivity of both sensors is sufficient for the environmental monitoring.

\section{Conclusion}

An analysis of graphene manufacturing methods has shown that the most promising technique for the production of electronic devices from this material is the thermolysis of a silicon carbide surface. However, films formed by this technique have lower carrier mobility than the films obtained by "exfoliation", but have predictable dimensions and are much more compatible with existing production methods for semiconductor devices. Simple resistive devices based on epitaxially-grown graphene were fabricated and tested for their sensitivity to $\mathrm{NO}_{2}$ gas. The devices are sensitive to low concentrations of $\mathrm{NO}_{2}$. The sensor based on the single-layer graphene exhibits a superior performance, compared with the analog that utilized multilayer graphene.

\section{Acknowledgements}

Author would like to thanks S. N. Novikov, S. P. Lebedev, Yu. N. Makarov, I. S. Kotousova, A. A. Lavrent'ev, I. V. Makarenko, V.N. Petrov, A. N. Smirnov, V. Yu. Davydov, S. Yu. Davydov, A. M. Strel'chuk,; D. V. Shamshur, N. V. Agrinskaya, V. A. Berezovets, V. I. Kozub, A. A. Sitnikova, R. Yakimova, V. M. Mikoushkin, V. V. Shnitov, S. Yu. Nikonov, T. Iakimov, O. Yu. Vilkov for help in experiments and fruitful discussion.

\section{References}

[1] Novoselov K.S., Geim A.K., et al. Electric field effect in atomically thin carbon films. Science, 2004, 306, P. 666 . 
[2] Geim A., Novoselov K. The rise of graphene. Nature Mater., 2007, 6, P. 183.

[3] Soldano C., Mahmood A., Dujardin E. Production, properties and potential of graphene. Carbon, 2010, 48, P. $2127-2150$.

[4] Wu Y.H., Yu T., Shen Z.X. Two-dimensional carbon nanostructures: fundamental properties, synthesis, characterization, and potential applications. J. Appl. Phys., 2010, 108, 071301 (1-38).

[5] Edwards R.S., Coleman K. Graphene synthesis: relationship to applications. Nanoscale, 2013, 5, P. $38-51$.

[6] Berger C., Song Z., et al. Ultrathin epitaxial graphite: 2D electron gas properties and a route toward graphenebased nanoelectronics. J. Phys. Chem. B, 2004, 108, P. 19912.

[7] Pearce R., Iakimov T., et al. Epitaxially grown graphene based gas sensors for ultra-sensitive $\mathrm{NO}_{2}$ detection. Sensors and Actuators B, 2011, 155, P. 451-455.

[8] Novikov S., Satrapinski A., Lebedeva N., Iisakka I. Sensitivity Optimization of Epitaxial Graphene-Based Gas Sensors. IEEE Trans. Instrum. Meas., 2013, 62 (6), P. 1859.

[9] Badami D.V. X-ray studies of graphite formed by decomposing silicon carbide. Carbon, 1965, 3, P. $53-57$.

[10] Kusunoki M., Suzuki T., et al. A formation mechanism of carbonnanotube films on SiC (0001). Appl. Phys. Lett., 2000, 77 (4), P. 531-533.

[11] Kusunoki M., Suzuki T., et al. Aligned carbon nanotube films on SiC (0001) wafers. Physica B, 2002, 303, P. 296-298.

[12] Rollings E., Gweon G.-H., et al. Synthesis and characterization of atomically-thin graphite films on a silicon carbide substrate. J. Phys. Chem. Solids, 2006, 67, P. 2172-2177.

[13] Emtsev K.V., Seyller Th., et al. Initial stages of the graphite-SiC(0001) interface formation studied by photoelectron spectroscopy. Mater. Sci. Forum, 2007, 556/557, P. 525-528.

[14] Hass J., Jeffrey C.A., et al. Highly-ordered graphene for 2D electronics. Appl. Phys. Lett., 2006, 89, 143106 (3 p).

[15] Lebedev S.P., Lebedev A.A., et al. Investigation of nanocarbon films on $\mathrm{SiC}$ surface Formed by sublimation in vacuum. Fuller., Nanotub. Carbon Nanostr., 2010, 18, P. 501-504.

[16] Lebedev S.P., Strel'chuk A.M., et al. Transport properties of multi-graphene films grown on semi-insulating SiC. Fuller., Nanotub. Carbon Nanostr., 2012, 20, P. 553-557.

[17] Seyller Th., Emtsev K.V., et al. Electronic structure of graphite/6H-SiC interfaces. Mater. Sci. Forum, 2007, 556-557, P. 701-704.

[18] Hass J., Feng R., et al. The structural properties of the multi-layer graphene/4H-SiC (0001) system as determined by surface X-ray diffraction. Phys. Rev. B, 2007, 75, 214109 (1-8).

[19] Ragan-Kelley B.L., Williams J.R., Friend C.M. Building a Metallic Graphene Transistor. Harvard DEAS REU Program, 2005, URL: www . yumpu.com/en/document/view/35107623/.

[20] Gu Gong, Nie Shu, et al. Field effect in epitaxial graphene on a silicon carbide substrate. Appl. Phys. Lett., 2007, 90, P. 253507.

[21] Kisoda K., Kamoi S., et al. Few epitaxial graphene grown on vicinal $6 \mathrm{H}-\mathrm{SiC}$ studied by deep ultraviolet Raman spectroscopy. Appl. Phys. Lett., 2010, 97, P. 033108.

[22] Peres N.M.R. The transport properties of grapheme: an introduction. arXiv:1007.2849v2.

[23] Lebedev A.A., Agrinskaya N.V., et al. Low temperature transport properties of grapheme and multigraphene structures on $6 \mathrm{H}-\mathrm{SiC}$ obtained by thermal graphitization: Evidence of a presence of nearly perfect Graphene Layer. J. Mater. Sci. Engin. A, 2013, 3 (11), P. 757-762.

[24] Lebedev A.A., Agrinskaya N.V., et al. Low temperature transport properties of graphene and multigraphene layers. Mater. Sci. Forum, 2013, 740-742, P. 137-140.

[25] Mikoushkin V.M., Shnitov V.V., et al. Size confinement effect in graphene grown on 6H-SiC (0001) substrate. Carbon, 2015, 86, P. 139-145.

[26] Hass J., de Heer W.A., Conrad E.H. The growth and morphology of epitaxial multilayer grapheme. J. Phys.: Condens. Matter, 2008, 20, P. 323202.

[27] Lebedev S.P. Petrov V.N., et al. Formation of periodic stepson 6H-SiC (0001) surface by annealing in a high vacuum. Mater. Sci. Forum, 2011, 679-680, P. 437-440.

[28] Ohta T., Bostwik A., et al. Interlayer interaction and electronic screening in multilayer graphene investigated with angle-resolved photoemission spectroscopy. Phys. Rev. Lett., 2007, 98, P. 206802.

[29] Virojanadara C., Yakimova R., Zakharov A.A., Johansson L.I. Large homogeneous mono-/bi-layer graphene on 6H-SiC (0001) and buffer layer elimination. J. Phys. D: Appl. Phys., 2010, 43, P. 374010.

[30] Becker T., Muhlberger S., et al. Air pollution monitoring using tin-oxide-based microreactor systems. Sensors and Actuators B, 2000, 69, P. 108-119. 